11

\title{
Квазистационарное приближение в задаче возбуждения низкочастотных электромагнитных полей в литосфере
}

\author{
() Е.Д. Терещенко, ${ }^{1}$ П.Е. Терещенко ${ }^{2}$ \\ ${ }^{1}$ Полярный геофизический институт, \\ 183010 Мурманск, Россия \\ ${ }^{2}$ Санкт-Петербургский фрилиал ИЗМИРАН им. Н.В. Пушкова, \\ 199034 Санкт-Петербург, Россия \\ e-mail: tereshchenko@gmail.com
}

Поступило в Редакцию 3 апреля 2020 г.

В окончательной редакции 13 мая 2020 г.

Принято к публикации 26 мая 2020 г.

Рассмотрено возбуждение горизонтальной заземленной антенной электромагнитного поля СНЧ-КНЧ и более низкого частотного диапазона в двуслойной среде. Получены приближенные аналитические формулы, достаточно полно описывающие поведение полей в низкочастотном диапазоне. Проанализирована точность использования квазистационарного приближения. Оценена возможность замены фактического поверхностного импеданса на импеданс плоской волны, нормально падающей на границу раздела. Для ряда экспериментов, проведенных на Кольском полуострове в рамках международного сотрудничества (FENICS-2014, FENICS-2019), определены частотные границы применимости приближенного подхода в оценке проводимости Земли.

Ключевые слова: КНЧ, распространение радиоволн; импеданс; электромагнитное поле; проводимость.

DOI: 10.21883/JTF.2021.01.50277.119-20

\section{Введение}

Введенное в работе [1] при вычислении электромагнитного поля у плоской границы раздела квазистационарное приближение в последующем широко использовалось в работах по электромагнитному зондированию Земли $[2,3]$. В его рамках сравнительно простые выражения, описывающие возбуждение низкочастотного электромагнитного поля, позволили связать поверхностный импеданс с импедансом плоской электромагнитной волны, а соответственно с проводимостью среды, что открыло возможность широкого применения низкочастотных полей как естественного [4], так и искусственного происхождения $[3,5]$ для глубинного зондирования структуры Земли.

Необходимым условием равенства двух упомянутых выше импедансов в квазистационарном приближении является размещение точки приема от источника на расстоянии в несколько скин-слоев для подстилающей среды. Это требование обратно ограничению, накладываемому квазистационарным приближением, для которого, наоборот, желательно, чтобы точка измерения находилась ближе к источнику. В частности, отметим, что при проводимости $5 \cdot 10^{-5} \mathrm{~S} / \mathrm{m}$ и частоте $2 \mathrm{~Hz}$ толщина скин-слоя равна $50 \mathrm{~km}$. В настоящей работе определим область, в которой имеется компромисс между этими требованиями и, соответственно, возможность использования импеданса плоской волны для определения проводимости Земли.

\section{1. Аналитическое приближение для полей линейного источника}

Рассмотрим простую модель, отражающую основные особенности рассматриваемой задачи. Считаем, что поле в двуслойной среде возбуждается питаемой током с гармонической зависимостью $\exp (-i \omega t)$ горизонтальной заземленной линейной антенной. Центр декартовых координат помещаем в середину антенны, ось $z$ направляем вверх, ось $x$ - вдоль антенны, а ось $y-$ поперек антенны. Расстояние на плоскости $(x, y, 0)$ от центра антенны обозначим $\rho$, а $\rho_{n}-$ расстояние от произвольной точки антенны. Среду в области $z>0$ предполагаем практически непроводящей $(\sigma=+0$, где наличие „+““ у нуля указывает на небольшое поглощение) с диэлектрической проницаемостью $\varepsilon_{0} \simeq 10^{-9} /(36 \pi) \mathrm{F} / \mathrm{m}$ и магнитной проницаемостью $\mu_{0}=4 \pi \cdot 10^{-7} \mathrm{H} / \mathrm{m}$. При этом область $z<0$ имеет электромагнитные параметры $\varepsilon, \mu_{0}, \sigma_{1}$.

Для определения компонент электромагнитного поля, возбуждаемого сторонним током $J$, как показано в [6], необходимо решить уравнение Гельмгольца для электрического вектора потенциала с соответствующими граничными условиями. Учитывая, что тангенциальные составляющие поля являются непрерывными функциями на границе $z=0$, воспользуемся результатами, приведенными в [6] для поля ниже границы раздела. Чтобы не загромождать дальнейший анализ, будем рассматривать две составляющие поля: $E_{y}$ и $H_{x}$. В соответствии с [6], для точечного горизонтального заземленного диполя, 
расположенного в центре координат, имеем

$E_{y}^{(1)}(\rho, z)=-\frac{J \Delta x}{2 \pi} i \omega \mu_{0} \frac{\partial}{\partial x} \frac{\partial}{\partial y} \int_{0}^{\infty} \frac{\exp \left(\nu_{1} z\right)}{\varkappa_{0}^{2} \nu_{1}+\varkappa_{1}^{2} \nu_{0}} J_{0}(\lambda \rho) \lambda d \lambda$,

где $J$ - ток, $J \Delta x-$ дипольный момент, $\Delta x-$ длина диполя, стремящаяся к бесконечно малой величине $\rho=\sqrt{x^{2}+y^{2}}, \varkappa_{j}=-i k_{j}, j=0,1$ указывает на среду, $k_{0} \simeq \omega / c, k_{1}=(\omega / c) \sqrt{\varepsilon_{1} / \varepsilon_{0}+i \sigma_{1} /\left(\omega \varepsilon_{0}\right)}, v_{j}=\sqrt{\varkappa_{j}^{2}+\lambda^{2}}$. При этом ветвь квадратного корня фиксирована, исходя из условия $\operatorname{Re} v_{j}>0$. $J_{0}(\lambda \rho)-$ функция Бесселя нулевого индекса.

Переход к полям, возбуждаемым линейной антенной длиной $2 L$, осуществляется суммированием полей диполей, расположенных по длине антенны. В частности отметим, что составляющая $\varepsilon_{y}$ электрического поля, возбуждаемая линейной антенной, будет равна

$$
\varepsilon_{y}=\sum E_{y}^{(j)}\left(\rho_{\eta}, z\right)=\left.\int_{-L}^{L} E_{y}^{(j)}\left(\rho_{\eta}, z\right)\right|_{J \delta x \rightarrow J} d \eta .
$$

Следовательно, основные закономерности в изменении $E_{y}^{(j)}(\rho, z)$ будут отражаться и в поле, возбуждаемом линейной антенной. Поэтому в дальнейшем удобно провести исследование, связанное с элементарным излучателем.

Магнитная составляющая поля $H_{x}^{(1)}(\rho, z)$ описывается выражением

$$
\begin{aligned}
H_{x}^{(1)}(\rho, z)= & -\frac{J \Delta x}{2 \pi} \frac{\partial}{\partial x} \frac{\partial}{\partial y} \int_{0}^{\infty} \frac{\left(\varkappa_{0}^{2}-\varkappa_{1}^{2}\right) \exp \left(v_{1} z\right)}{\left(\nu_{0}+v_{1}\right)\left(\varkappa_{0}^{2} v_{1}+\varkappa_{1}^{2} v_{0}\right)} \\
& \times J_{0}(\lambda \rho) \lambda d \lambda .
\end{aligned}
$$

Преобразуем формулы (1) и (3), выполнив дифференцирование по $y$ и введя новые безразмерные параметры $\tau_{0}=\rho \varkappa_{0}, \tau_{1}=\rho \varkappa_{1}$ и безразмерную переменную интегрирования $s=\lambda \rho$. В результате получим

$$
\begin{gathered}
E_{y}^{(1)}(\rho, z)=-\frac{J \Delta x}{2 \pi \varkappa_{1}} z_{g} \frac{\partial}{\partial x} \frac{y}{\rho^{3}} F_{E}(\rho, z), \\
H_{x}^{(1)}(\rho, z)=-\frac{J \Delta x}{2 \pi \varkappa_{1}} \frac{\partial}{\partial x} \frac{y}{\rho^{3}} F_{H}(\rho, z) .
\end{gathered}
$$

Здесь

$$
F_{E}(\rho, z)=r_{1}^{2} \int_{0}^{\infty} \frac{\exp \left(\sqrt{s^{2}+r_{1}^{2}} z / \rho\right)}{r_{0}^{2} \sqrt{s^{2}+r_{1}^{2}}+r_{1}^{2} \sqrt{s^{2}+r_{0}^{2}}} J_{1}(s) s^{2} d s,
$$

$F_{H}(\rho, z)=r_{1}\left(r_{1}^{2}-r_{0}^{2}\right)$

$$
\begin{aligned}
& \times \int_{0}^{\infty} \frac{\exp \left(\sqrt{s^{2}+r_{1}^{2}} z / \rho\right)}{\left(\sqrt{s^{2}+r_{1}^{2}}+\sqrt{s^{2}+r_{0}^{2}}\right)\left(r_{0}^{2} \sqrt{s^{2}+r_{1}^{2}}+r_{1}^{2} \sqrt{s^{2}+r_{0}^{2}}\right)} \\
& \times J_{1}(s) s^{2} d s,
\end{aligned}
$$

$J_{1}(s)$ - функция Бесселя с индексом, равным единице,

$$
z_{g}=\sqrt{\frac{\mu_{0}}{\varepsilon_{0}}} / \sqrt{\frac{\varepsilon_{1}}{\varepsilon_{0}}+i \frac{\sigma_{1}}{\omega \varepsilon_{0}}}
$$

- импеданс плоской монохроматической волны, нормально падающей на границу раздела [7].

Рассмотрим значения относительной диэлектрической проницаемости и проводимости для некоторых сред. Так, для морской воды ориентировочно $\varepsilon_{1} / \varepsilon_{0}=80$, а проводимость $\sigma=1 \mathrm{~S} / \mathrm{m}$, для сухой почвы $-\varepsilon_{1} / \varepsilon_{0} \sim 10$, а $\sigma=10^{-3} \mathrm{~S} / \mathrm{m}$, для мерзлотной почвы $\varepsilon_{1} / \varepsilon_{0} \sim 5$, а $\sigma=10^{-4} \mathrm{~S} / \mathrm{m}$. В ряде работ [4,5] отмечена очень низкая проводимость $\sim 5 \cdot 10^{-5} \mathrm{~S} / \mathrm{m}$ Мурманского блока на Кольском полуострове. В свете этого оценим величину $\sigma_{1} /\left(\omega \varepsilon_{0}\right) \simeq(\sigma / f) \cdot 1.8 \cdot 10^{10}$. Подставляя минимальное значение проводимости $5 \cdot 10^{-5}$, видим, что вплоть до частоты $f=100 \mathrm{kHz}$ эта величина будет значительно больше единицы. Поэтому для низкочастотного диапазона в выражении для волнового числа $k_{1}$ можно пренебречь вкладом относительной диэлектрической проницаемости на фоне слагаемого, содержащего проводимость, и считать, что

$$
k_{1} \simeq k_{0} \exp (i \pi / 4) \sqrt{\sigma_{1} /\left(\omega \varepsilon_{0}\right)},
$$

и соответственно

$$
z_{g}=\sqrt{-i\left(\omega \mu_{0} / \sigma_{1}\right)}
$$

Формулы (4), (5) позволяют найти поверхностный импеданс на границе раздела $z=0$ поля, возбуждаемого для антенны длиной $2 L$ :

$$
z_{y x}=\left.\frac{\varepsilon_{y}^{(0)}}{\mathscr{H}_{x}^{(0)}}\right|_{z=0}
$$

в которой $\mathscr{H}_{x}^{(0)}$ - магнитное поле, возбуждаемое линейной антенной. Вычисление $\mathscr{H}_{x}^{(0)}$ аналогично определению $\varepsilon_{y}^{(j)}$ по формуле (2).

Тангенциальные составляющие электромагнитного поля непрерывны на границе раздела, поэтому вместо поля в верхней среде в определение импеданса можно подставить горизонтальные составляющие поля в нижней среде и воспользоваться формулами (4), (5) для нахождения импеданса

$$
z_{y x}=z_{g} \frac{\left[\rho_{L}^{-3} F_{E}\left(\rho_{L}, 0\right)-\rho_{-L}^{-3} F_{E}\left(\rho_{-L}, 0\right)\right]}{\left[\rho_{L}^{-3} F_{H}\left(\rho_{L}, 0\right)-\rho_{-L}^{-3} F_{H}\left(\rho_{-L}, 0\right)\right]},
$$

где $\rho_{ \pm L}-$ расстояние до точки наблюдения от концов линейной антенны,

$$
F_{E}(\rho, 0)=\left.F_{E}(\rho, z)\right|_{z \rightarrow-0}, \quad F_{H}(\rho, 0)=\left.F_{H}(\rho, z)\right|_{z \rightarrow-0} .
$$

Из (8) следует, что при $F_{E}(\rho, 0)=F_{H}(\rho, 0)$ импеданс $z_{y x}$ будет равен импедансу плоской волны $z_{g}$.

Для того чтобы определить условия, необходимые для близости функций, рассмотрим их изменение как 
функций $r_{0}$. Начнем с квазистационарного приближения. Для него, согласно [1], полагают $\left|\varkappa_{0}\right|=0,\left|\varkappa_{1}\right| \neq 0$ или, в наших представлениях, $\left|r_{0}\right|=0$ при ненулевом значении $\left|r_{1}\right|$. Тогда из (4), (5) следует, что

$$
\left.\Phi_{E} \equiv F_{E}(\rho, 0)\right|_{\left|r_{0}\right|=0}=\left.\int_{0}^{\infty} \exp \left(\sqrt{s^{2}+r_{1}^{2}} z / \rho\right) J_{1}(s) s d s\right|_{z \rightarrow-0}
$$

$\left.\Phi_{H} \equiv F_{H}(\rho, 0)\right|_{\left|r_{0}\right|=0}=\left.r_{1} \int_{0}^{\infty} \frac{\exp \left(\sqrt{s^{2}+r_{1}^{2}} z / \rho\right)}{s+\sqrt{s^{2}+r_{1}^{2}}} J_{1}(s) s d s\right|_{z \rightarrow-0}$.

Способ вычисления интегралов подобного вида изложен в [6], поэтому, пропуская промежуточные математические преобразования, можно получить $\Phi_{E}=1$, $\Phi_{H}=r_{1} I_{1}\left(r_{1} / 2\right) K_{1}\left(r_{1} / 2\right)$. Здесь $I_{1} \quad$ и $K_{1}-$ цилиндрические функции мнимого аргумента [8]. При $\left|r_{1}\right| / 2 \gg 1$ можно воспользоваться асимптотическими представлениями функций Бесселя, для которых $I_{1}\left(r_{1} / 2\right) K_{1}\left(r_{1} / 2\right) \rightarrow 1 / r_{1}$, следовательно, $\Phi_{H} \rightarrow 1$. Таким образом, в рамках квазистационарного приближения поверхностный импеданс совпадает с импедансом плоской волны при условии $\left|r_{1}\right| / 2 \gg 1$. Заметим, что $r_{1}=(1-i) \rho / d_{s}$, где $d_{s}=1 /\left(\sqrt{\pi f \mu_{0}} \sigma_{1}\right)$ - толщина скин-слоя, в силу чего требование $\left|r_{1}\right| / 2 \gg 1$ эквивалентно тому, чтобы расстояние до точки измерения было много больше скин-слоя.

Прежде чем переходить к численным расчетам, получим еще одно приближение для функций $F_{E}$ и $F_{H}$, учитывающее, в отличие от квазистационарного приближения, ненулевое значение $\left|r_{0}\right|$.

Вместо условия $\left|\varkappa_{0}\right|=0$ положим в (1) более мягкое ограничение $\left|\varkappa_{0} / \varkappa_{1}\right| \ll 1$, что имеет место при возбуждении низкочастотных волн. Тогда, пренебрегая членом, имеющим порядок $\left|\varkappa_{0}^{2} / \varkappa_{1}^{2}\right|$, из (1) получаем

$$
\begin{aligned}
E_{y}^{0}(\rho, z \rightarrow-0) \simeq & -\frac{J \Delta x}{2 \pi \varkappa_{1}^{2}} i \omega \mu_{0} \frac{\partial}{\partial x} \frac{\partial}{\partial y} \int_{0}^{\infty} \frac{\exp \left(v_{1} z\right)}{v_{0}} \\
& \times\left. J_{0}(\lambda \rho) \lambda d \lambda\right|_{z \rightarrow-0} .
\end{aligned}
$$

Вычисляя интеграл по $\lambda$ и дифференцируя по $\rho$, находим

$$
E_{y}^{0}(\rho, z \rightarrow-0) \simeq-\frac{J \Delta x}{2 \pi \varkappa_{1}} z_{g} \frac{\partial}{\partial x} \frac{y}{\rho^{3}} \tilde{F}_{E}(\rho, 0),
$$

где $\tilde{F}_{E}(\rho, 0)=\left(1+r_{0}\right) \exp \left(-r_{0}\right)$.

При $\left|r_{0}\right| \rightarrow 0 \quad \tilde{F}_{E}(\rho, 0)=\Phi_{E}$, т. е. результат квазистационарного приближения. Отметим, что формула (9) получена при ограничении $\left|\varkappa_{0} / \varkappa_{1}\right| \ll 1$, что эквивалентно выполнению неравенства $\left|r_{1} / r_{0}\right| \gg 1$ или $r_{0}^{1 / 2} \ll \sqrt{120 \pi \sigma_{1} \rho}$, поэтому при больших значениях $\left|r_{0}\right|$ результаты заметно расходятся. Следовательно, в дальнейшем представляют интерес исследование в области $\left|r_{0}\right| \leq 1$, где можно ожидать практического совпадения величины поля с квазистационарным приближением, а также не рассматривать влияние сферичности Земли для рассматриваемого нами диапазона частот.

Аналогичные вычисления можно провести и для $H_{x}^{(1)}(\rho, z \rightarrow-0)$. Если воспользоваться в (3) условием, что $\left|\varkappa_{0}^{2} / \varkappa_{1}^{2}\right| \ll 1$, то можно написать следующее приближенное выражение для $H_{x}^{(1)}(\rho, z \rightarrow-0)$

$$
\begin{gathered}
\tilde{H}_{x}(\rho,-0)=\left.H_{x}^{(1)}(\rho, z \rightarrow-0)\right|_{\left|\chi_{0}^{2} / \varkappa_{1}^{2}\right| \ll 1} \\
=\frac{J \Delta x}{2 \pi} \frac{\partial}{\partial x} \frac{\partial}{\partial y} \int_{0}^{\infty} \frac{\exp \left(v_{1} z\right)}{\left(v_{0}+v_{1}\right) v_{0}} J_{0}(\lambda \rho) \lambda d \lambda .
\end{gathered}
$$

Вычисляя интеграл по $\lambda$, получим

$$
\begin{aligned}
& \tilde{H}_{x}(\rho,-0)=\frac{J \Delta x}{4 \pi} \frac{\partial}{\partial x} \frac{\partial}{\partial y}\left[I_{0}\left(\rho \frac{\varkappa_{1}-\varkappa_{0}}{2}\right)\right. \\
& \left.\times K_{0}\left(\rho \frac{\varkappa_{1}+\varkappa_{0}}{2}\right)+I_{1}\left(\rho \frac{\varkappa_{1}-\varkappa_{0}}{2}\right) K_{1}\left(\rho \frac{\varkappa_{1}+\varkappa_{0}}{2}\right)\right],
\end{aligned}
$$

где $I_{0}, K_{0}, I_{1}, K_{1}$ - цилиндрические функции мнимого аргумента [8].

Запишем $\tilde{H}_{x}(\rho,-0)$ в виде $(5)$

$$
\tilde{H}_{x}(\rho,-0)=-\frac{J \Delta x}{2 \pi \varkappa_{1}} \frac{\partial}{\partial x} \frac{y}{\rho^{3}} \tilde{F}_{H}(\rho,-0),
$$

для чего выполним дифференцирование по у в предыдущей формуле. В результате находим, что

$$
\begin{aligned}
\tilde{F}_{H}(\rho,-0)= & r_{1} I_{1}\left(\frac{r_{1}-r_{0}}{2}\right) K_{1}\left(\frac{r_{1}+r_{0}}{2}\right)+\frac{r_{1} r_{0}}{2} \\
& \times\left[I_{0}\left(\frac{r_{1}-r_{0}}{2}\right) K_{1}\left(\frac{r_{1}+r_{0}}{2}\right)\right. \\
& \left.+I_{1}\left(\frac{r_{1}-r_{0}}{2}\right) K_{0}\left(\frac{r_{1}+r_{0}}{2}\right)\right] .
\end{aligned}
$$

Функция $\quad \tilde{F}_{H}(\rho,-0) \quad$ при $\quad\left|r_{1}-r_{0}\right| \gg 1 \quad$ имеет следующее асимптотическое представление: $\tilde{F}_{H}(\rho,-0) \sim\left(1+r_{0}\right) \exp \left(-r_{0}\right)$. Таким образом, функция $\tilde{F}_{H}(\rho,-0)$ в широком диапазоне не зависит от $\left|r_{1}\right|$ и соответственно от $\rho \sigma_{1}$ и определяется только изменением $r_{0}$.

\section{2. Оценка границы применимости квазистационарного приближения}

Представим $r_{1}$ в виде

$$
r_{1}=r_{0}^{1 / 2} \sqrt{\frac{\sigma_{1} \rho}{c \varepsilon_{0}}}=r_{0}^{1 / 2} \sqrt{120 \pi \sigma_{1} \rho}
$$

Журнал технической физики, 2021, том 91, вып. 1 

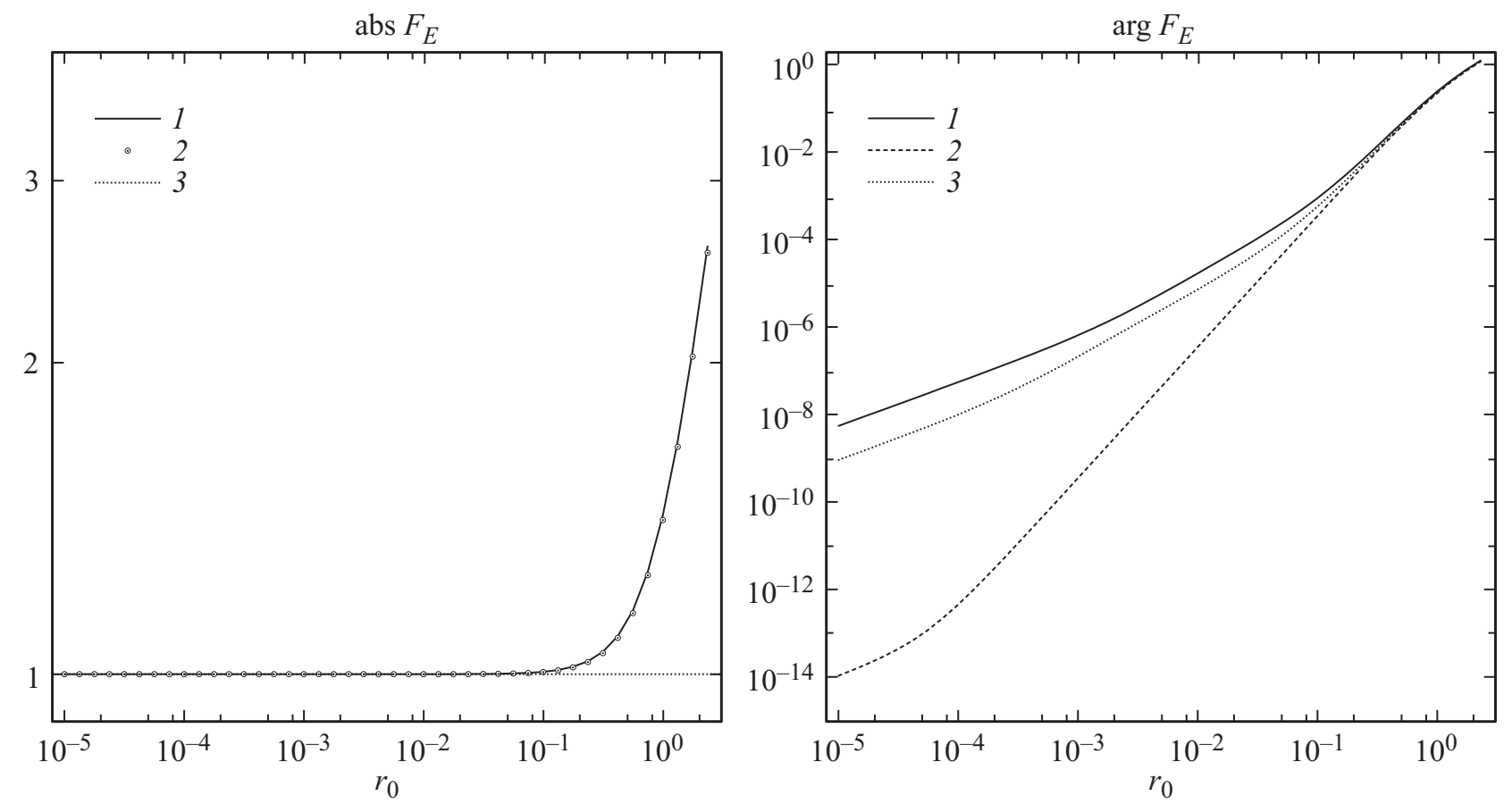

Рис. 1. Модуль и аргумент $F_{E}(\rho, 0)$, рассчитанные по формуле $(6)-1$, в квазистационарном приближении $\Phi_{E}-2$ и $F_{E}(\rho, 0)-3$.
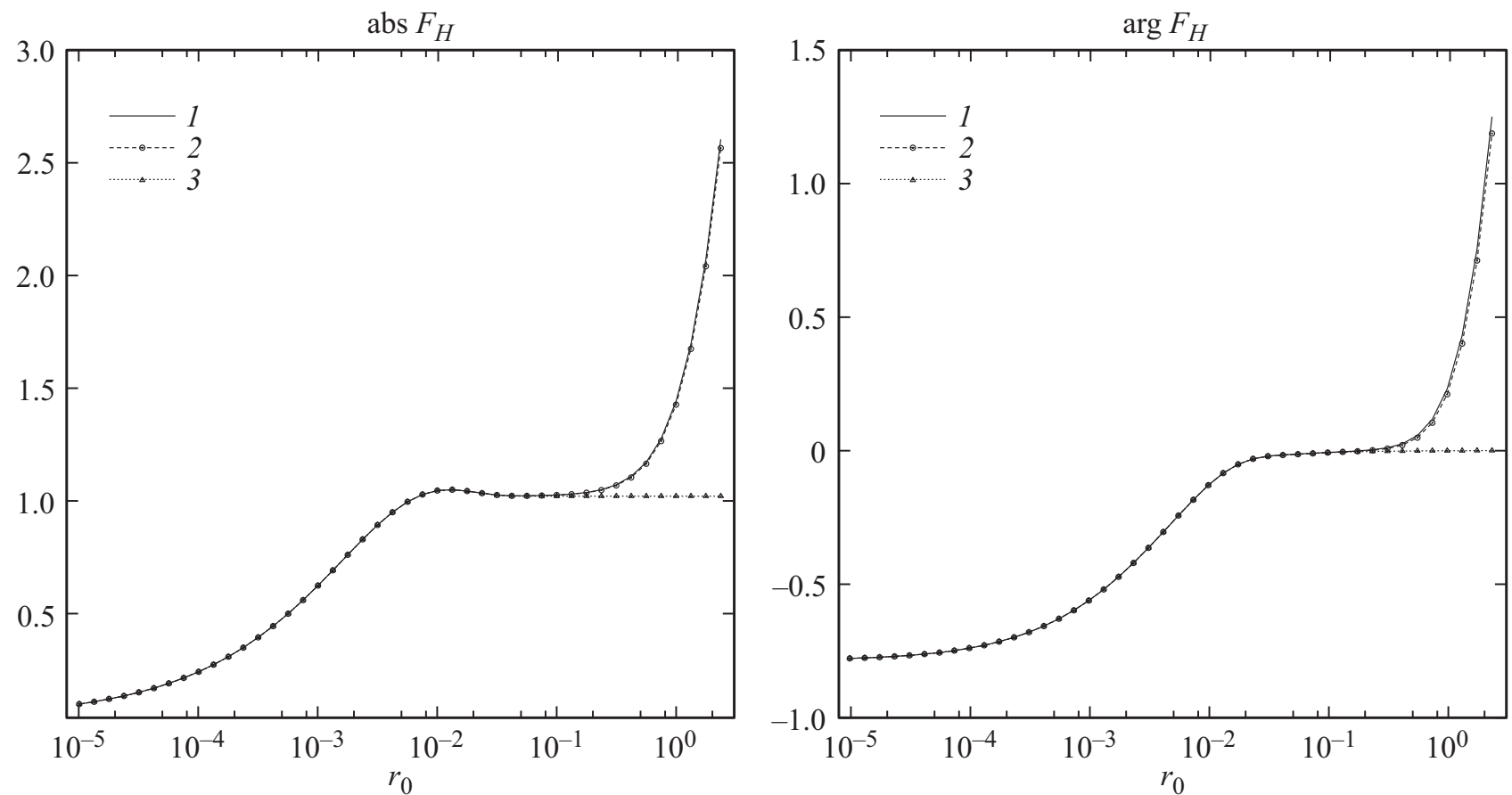

Рис. 2. Модуль и аргумент $F_{H}(\rho, 0)$, полученные по формуле $(7)-1$, в квазистационарном приближении $\Phi_{H}-2$ и функция $\tilde{F}_{H}(\rho, 0)-3$ при $\sigma_{1} \rho=5$.

и численно проанализируем, используя формулы (6) и (7), зависимость функций $F_{E}$ и $F_{H}$ от $\left|r_{0}\right|$ для различных значений параметра $\sigma_{1} \rho$.

На рис. 1 приведен результат расчета модуля и фазы функции $F_{E}$. Для сравнения на нем также нанесена функция $\tilde{F}_{E}$. Графики показывают хорошее совпадение функций $F_{E}$ и $\tilde{F}_{E}$. В то же время наблюдается их значительное отличие от единицы (квазистационарного приближения) по мере достижения $\left|r_{0}\right|$ единицы. Следующие графики (рис. 2, 3) относятся к сравнению результатов расчета $F_{H}$ по формуле из (5), в квазистационарном приближении $-\Phi_{H}$ и функции $\tilde{F}_{H}$. Здесь 

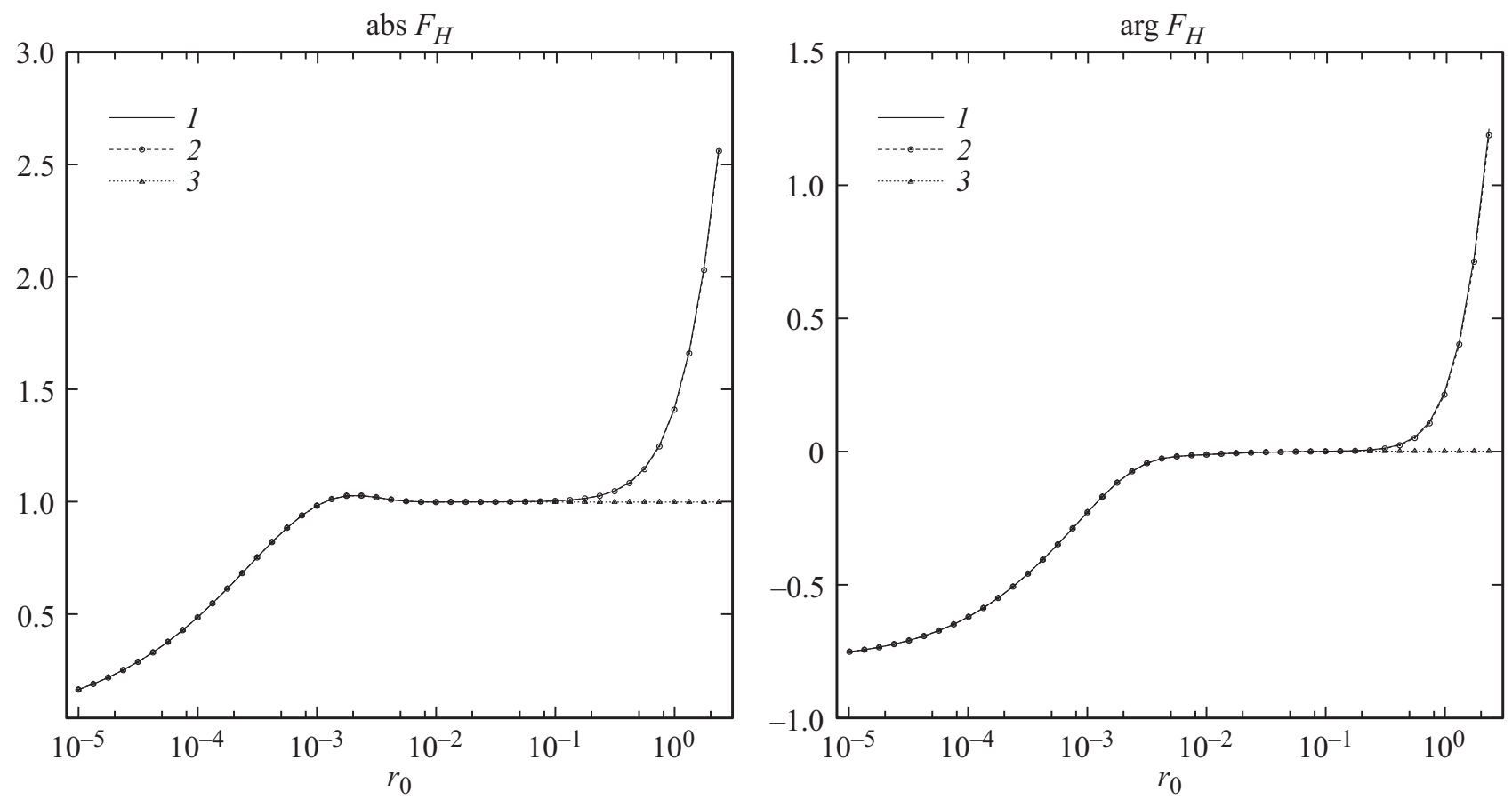

Рис. 3. Модуль и аргумент $F_{H}(\rho, 0)$, полученные по формуле $(7)-1$, в квазистационарном приближении $\Phi_{H}-2$ и функция $\tilde{F}_{H}(\rho, 0)-3$ при $\sigma_{1} \rho=30$.

наблюдается более сложная зависимость $F_{H}(\rho, 0)$ от $\left|r_{0}\right|$ : идет рост функции, затем она выходит на плато, после которого опять начинается рост. Расчеты показывают хорошее соответствие между функцией $F_{H}(\rho, 0)$ и ее приближением $\tilde{F}_{H}(\rho, 0)$ в большом диапазоне изменения $\left|r_{0}\right|$. При этом в квазистационарном приближении имеется только выход на плато со значением функции, равным единице. Область начиная с плато - это потенциальная область, где в рамках квазистационарного приближения поверхностный импеданс поля близок к импедансу плоской волны.

Как видим из рис. 2, 3, по мере уменьшения $\sigma_{1}$ при фиксированном $\rho$ длина плато сокращается и выход на него происходит при бо́льших значениях $\left|r_{0}\right|$, что увеличивает погрешность использования квазистационарного приближения. То есть низкая проводимость среды ограничивает снизу область частот, где применимо квазистационарное приближение. Ограничение частоты сверху происходит из-за параметра $\left|r_{0}\right|$, который по мере приближения к единице дает для $F_{E}$ результат, существенно отличающийся от квазистационарного приближения. Поэтому квазистационарное приближение применимо к описанию поля до значений $\left|r_{0}\right|$, значительно меньших единицы. Его точность в этой области имеет порядок $r_{0}^{2} / 2$. Более эффективной является аппроксимация функциями $\tilde{F}_{E}$ и $\tilde{F}_{H}$, справедливая до значений $\left|r_{0}\right|<120 \pi \sigma_{1} \rho$. Расчеты по этим функциям показывают, что для описания поверхностного импеданса импедансом плоской волны необходимо еще ограничение снизу $\left|r_{0}\right|>1 /\left(120 \pi \sigma_{1} \rho\right)$.
В то же время, опираясь на функции $\tilde{F}_{E}$ и $\tilde{F}_{H}$, можно утверждать, что, в отличие от полей, поверхностный импеданс совпадает с импедансом плоской волны в более широком, чем ограниченным квазистационарным приближением, интервале изменения параметра $\left|r_{0}\right|$. При $\left|r_{1}-r_{0}\right| \gg 1$, и равенство импедансов будет справедливо до $r_{0}$, ограниченных условием $\left|r_{1} / r_{0}\right| \gg 1$ или $\left|r_{0}\right| \ll 120 \pi \sigma_{1} \rho$.

В заключение рассмотрим применение результатов и выводов работы для экспериментов, выполненных в рамках проектов FENICS-2014 и FENICS-2019. Измерения на Кольском полуострове проводились в обсерватории Ловозеро в 2014 г. [9] и вблизи села Варзуга в 2019 г. [10]. Антенна находилась на севере Мурманской области, расстояние от концов антенны до точки измерений Ловозеро составило $100 \mathrm{~km}$ и $125 \mathrm{~km}$, до Варзуги $314 \mathrm{~km}$ и $370 \mathrm{~km}$. Рассмотрим две крайние ситуации с проводимостью $5 \cdot 10^{-5}$ и $5 \cdot 10^{-4}$. При этом первое значение подходит для описания эксперимента в 2014 г., а второе - для эксперимента в 2019 г. Диапазон частот, используемых в экспериментах, составлял $0.02-200 \mathrm{~Hz}$. Оценим возможность использования импеданса плоской волны для интерпретации экспериментальных данных. Для точки Ловозеро минимальное значение параметра $\sigma_{1} \rho$ равно пяти. В соответствии с рис. 2, область изменения $\left|r_{0}\right|$, в которой поверхностный импеданс равен импедансу плоской волны, определяется неравенством $\left|r_{0}\right| \geq 10^{-2}$. Используя значение $\left|r_{0}\right|$, зная $\rho$ и $\sigma_{1}$, получим ограничение по частоте $-f \geq 5 \mathrm{~Hz}$. Для точки Варзуга имеем $-f \geq 0.2 \mathrm{~Hz}$. По результатам из- 


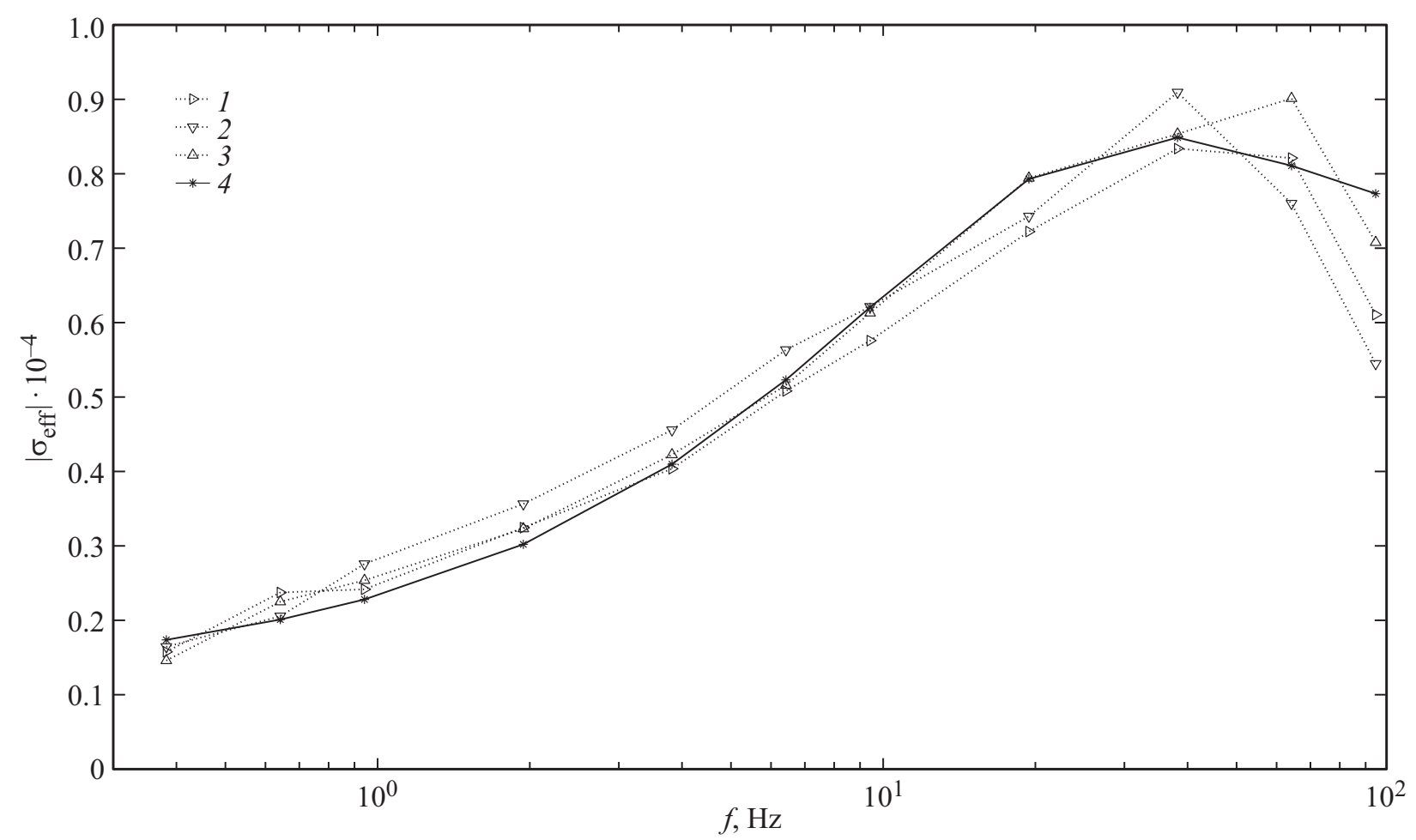

Рис. 4. Частотные зависимости модуля эффективной проводимости $\left|\sigma_{\text {eff }}\right|$, рассчитанные по экспериментальным данным $-1-3$ и для двухслойной модели -4 .

мерений амплитуд компонент электромагнитного поля монохроматического источника в точке Варзуга можно оценить эффективную проводимость литосферы во всем диапазоне использованных частот. Для эффективной проводимости плоской двуслойной структуры, используя подход, описанный в [7], получим

$$
\sqrt{\sigma_{\mathrm{eff}}}=\sqrt{\sigma_{1}} \frac{1+R_{12} \exp \left(-2 \varkappa_{1} d\right)}{1-R_{12} \exp \left(-2 \varkappa_{1} d\right)}
$$

где $\quad \varkappa_{1}=(1-i) \sqrt{\omega \mu_{0} \sigma_{1} / 2}, \quad R_{12}=\left(\sqrt{\sigma_{2}}-\sqrt{\sigma_{1}}\right)\left(\sqrt{\sigma_{1}}+\right.$ $\left.+\sqrt{\sigma_{2}}\right)$ ). Сравнение результатов модельных расчетов с экспериментально полученными (рис. 4, кривые $(1-3)$ показало, что оптимальными оказались следующие модельные параметры подстилающей поверхности: $\sigma_{1}=0.75 \cdot 10^{-4} \mathrm{~S} / \mathrm{m}, \sigma_{2}=10^{-5} \mathrm{~S} / \mathrm{m}, d=11.5 \mathrm{~km}$ (рис. 4, кривая 4).

Сравнивая параметры использованной модели с моделью электропроводности земной коры Фенноскандинавского щита и его обрамления из работы [5], можно сделать следующий вывод: используемые частоты не показывают неоднородности слоя литосферы глубже десяти километров. Для более детального изучения верхнего слоя, где наиболее заметно расхождение экспериментальных данных и модели, необходимо расширение частотного диапазона в сторону более высоких частот.

\section{Выводы}

Использование более мягкого ограничения (длина волны в вакууме много больше длинны волны в нижнем полупространстве) по сравнению с квазистационарным приближением, введенным в [1] и послужившим обоснованием для применения импедансного подхода при исследовании структуры литосферы [2,3], позволило оценить границы применимости квазистационарного приближения как для описания полей, так и поверхностного импеданса.

Найдены условия, при выполнении которых, возможна замена поверхностного импеданса на импеданс плоской волны.

Полученные в работе аналитические аппроксимации для электромагнитного поля, возбуждаемого горизонтальной заземленной антенной в СНЧ-КНЧ и более низком диапазоне, хорошо совпадают с точными численными расчетами.

Проанализирована ограниченность квазистационарного приближения и оценены границы его применения для описания результатов измерений электромагнитных полей в различных частях Кольского полуострова в рамках экспериментов $F_{E}$ NICS-2014 и $F_{E}$ NICS-2019. Показано, что в рамках измерений, проведенных на юго-востоке Кольского полуострова, квазистационарное приближение не работает при описании полей во всем диапазоне использованных частот $(0.02-95 \mathrm{~Hz})$, однако возможна 
замена поверхностного импеданса на импеданс плоской волны.

На основе импедансного подхода получены результаты по структуре проводимости литосферы в юго-восточной части Кольского полуострова, не противоречащие существующим геологическим данным. Финансирование работы Работа выполнена при поддержке Российского фонда фундаментальных исследований (проект № 19-05-00823).

\section{Конфликт интересов}

Авторы заявляют, что у них нет конфликта интересов.

\section{Список литературы}

[1] V. Fock. Annalen Der Physik, 409(4), 401 (1933). DOI: 10.1002/andp.19334090405

[2] А.В. Вешев. Электропрофилирование на постоянном $u$ переменном токе. 2 издание, переработанное $u$ дополненное. (Недра, 1980).

[3] Л.Л. Ваньян. Электромагнитные зондирования. (Научный мир, М., 1997).

[4] А.А. Ковтун. Строение коры и верхней мантии на северо-западе Восточно-Европейской платформы по данным магнитотеллурического зондирования. (Из-во ЛГУ, 1989).

[5] A.A. Zhamaletdinov, A.N. Shevtsov, E.P. Velikhov, A.A. Skorokhodov, V.E. Kolesnikov, T.G. Korotkova, P.A. Ryazantsev, B.V. Efimov, V.V. Kolobov, M.B. Barannik, P.I. Prokopchuk, V.N. Selivanov, Yu.A. Kopytenko, E.A. Kopytenko, V.S. Ismagilov, M.S. Petrishchev, P.A. Sergushin, P.E. Tereshchenko, B.V. Samsonov, M.A. Birulya, M.Yu. Smirnov, T. Korja, Yu.M. Yampolski, A.V. Koloskov, N.A. Baru, S.V. Poljakov, A.V. Shchennikov, G.I. Druzhin, W. Jozwiak, J. Reda, Yu.G. Shchors. Izvestiya, Atmospheric and Oceanic Physics, 51 (8), 826 (2015). DOI: $10.1134 / \mathrm{s} 0001433815080083$

[6] Е.Д. Терещенко, П.Е. Терещенко. Радиотехника и электроника, 63 (4), 1 (2018). DOI: 10.7868/S0033849418040034 E.D. Tereshchenko, P.E. Tereshchenko. Journal of Communications Technology and Electronics, 63(4), 335 (2018). DOI: 10.1134/S1064226918040125]

[7] Г. И. Макаров, В. В. Новиков, С.Т. Рыбачек. Распространение электромагнитных волн над земной поверхностью. (Наука, М.,1991).

[8] И.С. Градштейн, И.М. Рыжик. Таблицы интегралов, сумм, рядов и произведений. (Физматгиз, М., 1962).

[9] Е.Д. Терещенко, А.Е. Сидоренко, П.Е. Терещенко. ЖТФ, 2019, 89 (7), 1098 (2019)

DOI: 10.21883/JTF.2019.07.47805.388-18

[E.D. Tereshchenko, A.E. Sidorenko, P.E. Tereshchenko. Tech. Phys., 64 (7), 1029 (2019).

DOI: $10.1134 / \mathrm{S} 1063784219070259]$

[10] П.Е. Терещенко. Наука и технологические разработки, 4, 34 (2019). DOI: $10.21455 /$ std2019.4-3 